\title{
openheart Heart rate and adverse outcomes in patients with prevalent atrial fibrillation
}

\author{
Giorgio Moschovitis (D) ,,2 Linda S B Johnson (D) ,2,3 Steffen Blum, ${ }^{4,5}$ \\ Stefanie Aeschbacher (D) , ${ }^{4,5}$ Maria Luisa De Perna, ${ }^{1}$ Alberto Pagnamenta, ${ }^{6}$ \\ Patrizia Assunta Mayer Melchiorre, ${ }^{1}$ Alexander P Benz, ${ }^{2}$ Richard Kobza, ${ }^{7}$ \\ Marcello Di Valentino, ${ }^{8,9}$ Christine S Zuern, ${ }^{4,5}$ Angelo Auricchio, ${ }^{10}$ \\ Giulio Conte (D) , ${ }^{10}$ Nicolas Rodondi, ${ }^{11,12}$ Manuel R Blum, ${ }^{11,12}$ Juerg H Beer, ${ }^{13,14}$ \\ Michael Kühne, ${ }^{4,5}$ Stefan Osswald, ${ }^{4,5}$ David Conen, ${ }^{2}$ BEAT-AF and Swiss-AF \\ investigators
}

\begin{abstract}
- Additional supplemental material is published online only. To view, please visit the journal online (http://dx.doi.org/10. 1136/openhrt-2021-001606).
\end{abstract}

To cite: Moschovitis G, Johnson LSB, Blum S, et al. Heart rate and adverse outcomes in patients with prevalent atrial fibrillation. Open Heart 2021;8:e001606. doi:10.1136/ openhrt-2021-001606

GM and LSBJ contributed equally.

GM and LSBJ are joint first authors.

Received 1 February 2021 Revised 18 March 2021 Accepted 6 April 2021
Check for updates

(c) Author(s) (or their employer(s)) 2021. Re-use permitted under CC BY-NC. No commercial re-use. See rights and permissions. Published by BMJ.

For numbered affiliations see end of article.

Correspondence to Dr David Conen; david.conen@ phri.ca

\section{ABSTRACT}

Objective The optimal target heart rate in patients with prevalent atrial fibrillation (AF) is not well defined. The aim of this study was to analyse the associations between heart rate and adverse outcomes in a large contemporary cohort of patients with prevalent AF.

Methods From two prospective cohort studies, we included stable AF outpatients who were in AF on the baseline ECG. The main outcome events assessed during prospective follow-up were heart failure hospitalisation, stroke or systemic embolism and death. The associations between heart rate and adverse outcomes were evaluated using multivariable Cox regression models.

Results The study population consisted of 1679 patients who had prevalent AF at baseline. Mean age was 74 years, and $24.6 \%$ were women. The mean heart rate on the baseline ECG was $78( \pm 19)$ beats per minute $(\mathrm{bpm})$. The median follow-up was 3.9 years (IQR 2.2-5.0). Heart rate was not significantly associated with heart failure hospitalisation (adjusted HR (aHR) per $10 \mathrm{bpm}$ increase, $1.00,95 \% \mathrm{Cl} 0.94$ to $1.07, \mathrm{p}=0.95$ ), stroke or systemic embolism (aHR $0.95,95 \% \mathrm{Cl} 0.84$ to $1.07, \mathrm{p}=0.38$ ) or death (aHR 1.02, 95\% $\mathrm{Cl} 0.95$ to $1.09, \mathrm{p}=0.66$ ). There was no evidence of a threshold effect for heart rates $<60 \mathrm{bpm}$ or $\geq 100 \mathrm{bpm}$

Conclusions In this large contemporary cohort of outpatients with prevalent AF, we found no association between heart rate and adverse outcome events. These data are in line with recommendations that strict heart rate control is not needed in otherwise stable outpatients with AF.

\section{INTRODUCTION}

Atrial fibrillation (AF) is the most common sustained cardiac arrhythmia in the general population, and its prevalence is rapidly increasing. ${ }^{1} \mathrm{AF}$ is associated with an increased risk of heart failure, stroke and death. ${ }^{2-4}$

Treatment options for AF patients with prevalent $\mathrm{AF}$ on the ECG include both rhythm and rate control, as neither has been shown to be superior for patients eligible for

\section{Key questions}

What is already known about this subject?

- Heart rate control is considered first-line therapy option in patients with atrial fibrillation (AF), however, for the majority of stable outpatients with AF the optimal heart rate target is still unclear.

What does this study add?

- In this large contemporary cohort of patients with prevalent $A F$, heart rate assessed using a 10 s resting ECG was not associated with heart failure hospitalisation, stroke or systemic embolism and death. These data reinforce current recommendations that strict heart rate control is not needed to guide management in otherwise stable outpatients with AF.

How might this impact on clinical practice?

- In daily clinical practice, a large proportion of patients with AF are stable and asymptomatic. Our findings derived from a large community-based cohort will help patients and clinicians to guide decisions regarding heart rate control in patients with prevalent AF.

both options. ${ }^{5-8}$ While an increased heart rate has been consistently associated with mortality and morbidity in sinus rhythm patients, ${ }^{8-11}$ much less information is available on the prognostic value of heart rate in patients with prevalent AF. In the relatively small Rate Control Efficacy in Permanent Atrial Fibrillation: a Comparison between Lenient versus Strict Rate Control II (RACEII) trial, lenient rate control was not inferior to strict rate control for preventing the primary composite outcome of cardiovascular death, hospitalisation for heart failure, stroke, systemic embolism, bleeding and lifethreatening arrhythmic events in patients with permanent AF. ${ }^{6}$ Pooled data from RACE and AFFIRM did not show a significant difference in the primary endpoint (composite of 
mortality, cardiovascular hospitalisation and myocardial infarction) between a more strict (target heart rate $\leq 80$ beats per minute $(\mathrm{bpm})$ ) vs a more lenient (heart rate $<100 \mathrm{bpm}$ ) rate control. ${ }^{7}$ In another analysis, heart rate during AF was not associated with mortality, in contrast to heart rate during sinus rhythm. ${ }^{8}$

Current guidelines propose an initial resting heart rate target of $<110 \mathrm{bpm}$ mainly to improve haemodynamic stability, reduce symptoms and preserve left ventricular function. ${ }^{12}$ However, for the large group of AF patients who are haemodynamically stable and mostly asymptomatic, more data are needed to determine the optimal heart rate target.

We, therefore, conducted the current analysis to assess the associations between heart rate and several adverse outcome events in a large cohort of contemporary outpatients treated in standard care with prevalent AF during the baseline examination.

\section{MATERIALS AND METHODS Patient population}

The study population was derived from two similar and ongoing prospective cohort studies: Swiss AF (Swiss-AF, $\mathrm{n}=2415$ ) and Basel AF (BEAT-AF, $\mathrm{n}=1546$ ) cohort study. We excluded subjects who were not in AF or atrial flutter at the time of the baseline examination $(n=2207)$ or subjects with missing data on heart rhythm $(n=27)$ or other covariates $(n=48)$. The final study population consisted of 1679 individuals with $\mathrm{AF}$ at the baseline examination.

Written informed consent has been obtained from the participants (or their guardians).

Methodology and data collection in Swiss-AF and BEAT-AF were very similar and have been previously described in detail. ${ }^{13-16}$ Both studies have recruited from multiple sites in Switzerland, with enrolment during 2010-2014 for BEAT-AF and 2014-2017 for Swiss-AF. In both cohorts, patients had to be 65 years or older and have established, previously documented AF. A convenience sample of patients aged 45-64 years was enrolled in both cohorts to evaluate the effects of AF on the active workforce. Patient with an acute illness in the past 4 weeks could only be enrolled after clinical stabilisation. Patients with transient $\mathrm{AF}$ in connection with an acute illness or surgery were excluded. Patients enrolled in BEAT-AF could not participate in Swiss-AF.

\section{Baseline measurements and follow-up assessments}

Baseline examination included the same case report form in both cohorts. Heart rhythm and rate were obtained from a standard resting 12-lead ECG (BEAT-AF) or 16-lead ECG (Swiss-AF) during a 10 s registration. ${ }^{15}$ Weight $(\mathrm{kg})$ and height (metre $(\mathrm{m})$ ) were directly measured. Body mass index (BMI) was calculated as $\mathrm{kg} / \mathrm{m}^{2}$. Smoking status was categorised into current smokers, former smokers and never smokers. Regular physical activity was defined as physical exercise on a regular basis (yes/no). Alcohol consumption was calculated as number of standard unit drinks per day. AF was classified according to the 2010 guidelines of the European Society of Cardiology into paroxysmal (self-terminating), persistent (lasting $\geq 7$ days or requiring termination by electrical or pharmacological cardioversion) or permanent AF (accepted by patient and physician).${ }^{17}$ Antiarrhythmic drugs were categorised according to the Vaughan-Williams classification: class 1 (sodium-channel blocker), class 2 (beta-blockers), class 3 (amiodarone, sotalol and dronedarone) and class 4 (non-dihydropyridine calcium-channel blocker). History of coronary artery disease (CAD) was defined as a history of myocardial infarction, percutaneous coronary angioplasty or coronary by-pass graft surgery. After an in-person baseline visit in both cohorts, follow-up was conducted annually by mail and phone interviews in BEAT-AF, and by in-person visits in Swiss-AF.

\section{Clinical outcome events}

Both cohorts used the same definitions for all clinical adverse outcome events. All events were validated by two physicians in a standardised way, on the basis of all available information. In case of discordant assessments, a third reviewer physician was consulted. Incident heart failure hospitalisation was defined as a hospitalisation for congestive heart failure that was associated with at least one overnight stay, or a congestive heart failure exacerbation that led to an extension of an existing hospitalisation. Stroke was defined as an acute focal neurological deficit of vascular origin, confirmed by imaging (CT or MRI) or autopsy, and systemic embolism was defined as evidence of abrupt occlusion of a systemic artery consistent with an embolic event. Detailed outcome event definitions are provided in online supplemental table 1 .

\section{Patient and public involvement}

Patients and public were not involved in the design, or conduct, or reporting, or dissemination plans of this research.

\section{Statistical analysis}

Baseline characteristics are presented across quartiles of heart rate. Differences across quartiles were analysed using analysis of variance for normally distributed continuous variables, Kruskal-Wallis tests for non-normally distributed continuous variables and $\chi^{2}$ tests for categorical variables.

Follow-up analyses to evaluate the associations between heart rate and clinical outcome events were performed using Cox regression models adjusted for two prespecified set of covariates. In model 1, we adjusted for age and sex. In model 2, we additionally adjusted for BMI, history of hypertension, history of diabetes mellitus, history of CAD, history of stroke or transient ischaemic attack, history of heart failure, type of AF (paroxysmal and persistent vs permanent), smoking status, alcohol consumption, physical activity, use of antiarrhythmic medication according to the Vaughan Williams classification, digoxin and oral 
anticoagulation. The associations between heart rate and outcome events were analysed per $10 \mathrm{bpm}$ as well as by prespecified categories for low heart rate defined as $<60 \mathrm{bpm}$, or high heart rate defined as $\geq 100 \mathrm{bpm}$.

The shape of the association of heart rate with the heart failure hospitalisation, stroke and mortality was further assessed by including a quadratic term into model 2. Interaction between heart rate and sex or history of heart failure at baseline was assessed by including a multiplicative interaction parameter in model 2. Finally, we further assessed the association between heart rate and heart failure hospitalisation and stroke or systemic embolism after accounting for the competing risk of mortality using competing risk regression as described by Fine and Gray. ${ }^{18}$ The proportional hazards assumption was assessed visually using Nelson-Aalen plots and found to be valid for all models. A two-sided $p<0.05$ was considered statistically significant. All analyses were performed with Stata SE V. 15.1. Data are not available.

\section{RESULTS}

The mean age of the study population $(n=1679)$ was 74 years (SD 9 years), $24.6 \%$ were women, and $51.4 \%$ had permanent AF. The mean heart rate at the time of enrollment was 78 bpm (SD 19 bpm, range 31-160 bpm); $9.7 \% \quad(\mathrm{n}=162)$ had a heart rate $<60 \mathrm{bpm}$, and $12.0 \%$ $(\mathrm{n}=201)$ a heart rate $\geq 100 \mathrm{bpm}$. The median follow-up duration was 3.9 years (IQR 2.2-5.0). Baseline characteristics stratified by quartiles of heart rate are reported in table 1 .

\section{Heart rate and heart failure hospitalisation}

During the follow-up, there were 301 heart failure hospitalisations, corresponding to an incidence rate of 47.4 (95\% CI 42.3 to 53.0) per 1000 person-years. There was no significant association between heart rate and heart failure hospitalisation using either Cox regression models or competing risks regression (tables 2 and 3). We found no evidence of a nonlinear association ( $p$ for quadratic term $=0.47$ ), nor was there a significant association between heart rates $<60$ or $\geq 100 \mathrm{bpm}$ with heart failure hospitalisation (table 2). There was no evidence of interaction between heart rate and either sex ( $p$ for interaction $=0.99$ ) or history of heart failure ( $p$ for interaction $=0.22$ ).

\section{Heart rate and stroke or systemic embolism}

There were 96 stroke or systemic embolism events during follow-up, corresponding to an incidence rate of 14.4

\begin{tabular}{|c|c|c|c|c|c|}
\hline Characteristics ( $n=1679)$ & Quartile $1(n=395)$ & Quartile $2(n=442)$ & Quartile 3 ( $n=395)$ & Quartile $4(n=447)$ & $P$ value \\
\hline Heart rate range, bpm & $31-64$ & $65-75$ & $76-86$ & $87-160$ & \\
\hline Age, years & $75.5(8.5)$ & $75.0(8.6)$ & $73.9(8.8)$ & $71.9(9.6)$ & $<0.0001$ \\
\hline Female sex, \% & 21.8 & 21.5 & 26.6 & 28.6 & 0.03 \\
\hline Body mass index, $\mathrm{kg} / \mathrm{m}^{2}$ & $27.5(4.6)$ & $28.0(4.9)$ & $28.0(4.8)$ & $28.0(4.9)$ & 0.37 \\
\hline History of coronary artery disease, \% & 38.7 & 33.3 & 28.4 & 22.2 & $<0.0001$ \\
\hline History of stroke or TIA, \% & 22.5 & 19.5 & 21.8 & 17.2 & 0.21 \\
\hline History of hypertension, \% & 76.5 & 76.9 & 73.2 & 75.6 & 0.61 \\
\hline History of heart failure, \% & 39.5 & 31.9 & 33.9 & 28.6 & 0.009 \\
\hline History of diabetes, \% & 21.0 & 19.2 & 18.5 & 18.8 & 0.81 \\
\hline \multicolumn{6}{|l|}{ Smoking status, \% } \\
\hline Current & 5.6 & 6.6 & 6.3 & 9.0 & 0.20 \\
\hline Former & 53.2 & 50.5 & 45.8 & 48.8 & \\
\hline Never & 41.3 & 43.0 & 47.9 & 42.3 & \\
\hline Oral anticoagulation, \% & 93.9 & 92.5 & 94.7 & 88.1 & 0.002 \\
\hline \multicolumn{6}{|l|}{ Antiarrhythmic drugs*, \% } \\
\hline Class $1 \mathrm{c}$ & 1.0 & 1.1 & 1.0 & 2.5 & 0.20 \\
\hline Class 2 & 65.8 & 74.9 & 75.2 & 75.2 & 0.004 \\
\hline Class 3 & 8.1 & 10.6 & 11.7 & 14.3 & 0.04 \\
\hline Class 4 & 27.6 & 26.0 & 18.2 & 18.6 & 0.001 \\
\hline Use of digoxin, \% & 9.1 & 9.1 & 8.9 & 7.2 & 0.69 \\
\hline Regular physical activity, \% & 42.4 & 42.5 & 42.5 & 43.2 & 0.99 \\
\hline Alcohol consumption, standard units/day & $0.80(1.4)$ & $0.57(1.4)$ & $0.43(1.2)$ & $0.50(1.1)$ & 0.13 \\
\hline
\end{tabular}

Normally distributed continuous data are presented as means (SD), qualitative data are presented as absolute numbers (percentages). Non-normally distributed data (alcohol use) are presented as median (IQR).

*Vaughan William classifications: class 1c, sodium-channel blockers, class 2=beta-blockers, class $3=$ =amiodarone, sotalol and dronedarone; class $4=$ non-dihydropyridine calcium-channel blockers.

TIA, transient ischaemic attack. 
Table 2 Cox regression models for the associations of resting heart rate with adverse outcomes

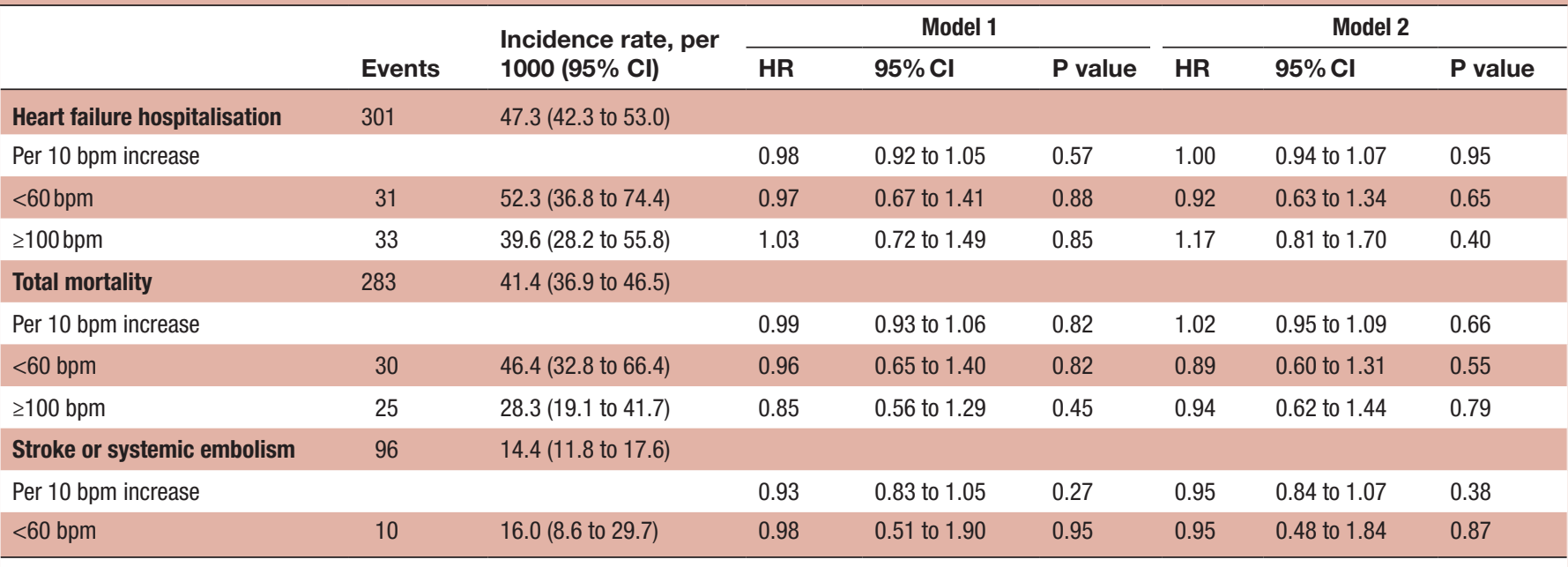

Model 1 is adjusted for age and sex.

Model 2 is adjusted for model 1+body mass index, physical activity, smoking status (never, former or current), type of AF (paroxysmal and persistent vs permanent), alcohol use (log-transformed), history of stroke/TIA, history of heart failure, hypertension, diabetes, the use of beta-blockers, nondihydropyridine calcium-channel blockers, class 1c antiarrhythmic drugs, class 3 antiarrhythmic drugs, digoxin and oral anticoagulation.

$\mathrm{AF}$, atrial fibrillation; TIA, transient ischaemic attack.

(95\% CI 11.8 to 17.6 ) per 1000 person-years. We found no evidence of an association between heart rate and stroke or systemic embolism, in either Cox or competing risks models (tables 2 and 3). The number of events among subjects with high heart rates was very small $(n=3)$, and associations with stroke were not assessed in this stratum. There was no evidence of a nonlinear relationship $(p=0.30)$. We found no evidence of interaction between sex and heart rate ( $p$ for interaction $=0.85$ ) or history of heart failure and heart rate ( $p$ for interaction $=0.11$ ).

\section{Heart rate and mortality}

There were 283 deaths during follow-up (incidence rate 41.4 (95\% CI 36.9 to 46.5 ) per 1000 person-years. Heart rate was not associated with mortality, either as a continuous variable or among subjects with low or high heart rates (table 2), and there was no evidence of a nonlinear association $(\mathrm{p}=0.20)$. We found no evidence of an interaction between heart rate and either sex ( $p$ for interaction $=0.77$ ) or history of heart failure status ( $p$ for interaction $=0.17$ ).

\section{DISCUSSION}

In this large cohort of stable outpatients with prevalent $\mathrm{AF}$ at the time of enrolment, we found no significant associations of heart rate with the risks of incident heart failure hospitalisation, stroke or systemic embolism and death. Most patients had a resting heart rate between 60 and $100 \mathrm{bpm}$, likely a reflection of the inclusion of stable outpatients in this contemporary cohort, who would have received treatment if they had more extreme heart rates. There was no evidence of nonlinear associations, and neither a heart rate $<60 \mathrm{bpm}$ nor a heart rate $\geq 100 \mathrm{bpm}$ was significantly associated with adverse outcomes.

Table 3 Competing risks regression analyses for incident heart failure and stroke by heart rate

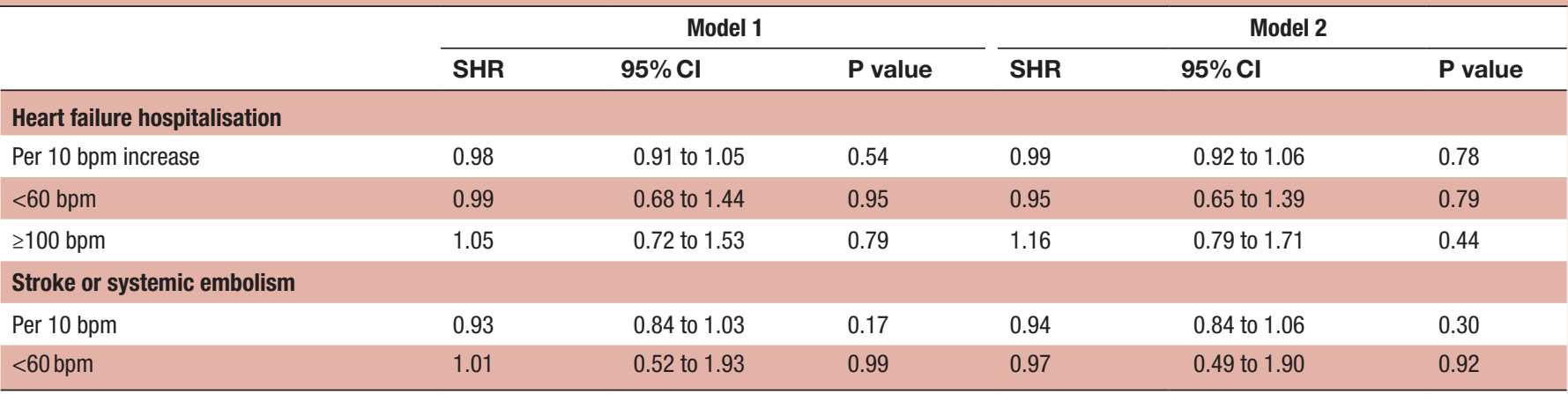

Model 1 is adjusted for age and sex.

Model 2 is adjusted for model $1+$ body mass index, physical activity, smoking status (never, former or current), type of AF (paroxysmal and persistent vs permanent), alcohol use (log-transformed), history of stroke/TIA, history of heart failure, hypertension, diabetes, the use of beta-blockers, nondihydropyridine calcium-channel blockers, class 1c antiarrhythmic drugs, class 3 antiarrhythmic drugs, digoxin and oral anticoagulation. Includes 1679 subjects, with 162 subjects with heart rate $<60$ and 201 subjects with heart rate $\geq 100 \mathrm{bpm}$.

AF, atrial fibrillation; SHR, sub-HR; TIA, transient ischaemic attack. 
The Early Treatment of Atrial Fibrillation for Stroke Prevention Trial (EAST-AFNET) recently found that early rhythm control treatment improved adverse clinical outcomes in patients with early AF. ${ }^{19}$ Whether these data apply to a wider group of AF patients is currently unclear. In addition, in symptomatic patients with uncontrolled $\mathrm{AF}$, rate or rhythm control are important to improve haemodynamic stability and AF-related symptoms and to prevent or reverse systolic dysfunction. ${ }^{32}{ }^{12}{ }^{20}$ However, in daily clinical practice, the great majority of elderly patients with AF do not complain about AF-related symptoms and are haemodynamically stable, despite widely varying heart rates, and despite variable use of rate-controlling agents. Our data do not apply to the first group of patients, but do suggest that in the latter population, more stringent heart rate control is not needed for the prevention of adverse outcomes. The present study suggests that this concept is applicable to a broad group of elderly patients with a high prevalence of comorbidities.

In previous studies, there has been some controversy regarding the prognostic significance of heart rate. For example, a higher heart rate on the baseline ECG was associated with incident heart failure in a study where patients in $\mathrm{AF}$ and sinus rhythm were analysed together. ${ }^{21}$ On the other hand, heart rate during AF was not associated with all-cause or cardiovascular mortality in a pooled analysis of two cohorts, in contrast to heart rate during sinus rhythm. ${ }^{8}$ Similarly, in patients with prevalent $\mathrm{AF}$, heart rate was not associated with the composite endpoint of death, cardiovascular hospitalisation and myocardial infarction after multivariable adjustment. ${ }^{7}$ Our study adds to this literature, by confirming that in stable contemporary outpatients with prevalent AF, heart rate was not associated with adverse outcomes. In addition, we found no evidence of a threshold effect for heart rates $<60 \mathrm{bpm}$ or $>100 \mathrm{bpm}$, considered adequate heart rate thresholds by current guidelines. ${ }^{22}$

Our data are in line with the RACE-II trial, where a strict rate control strategy $(<80 \mathrm{bpm}$ at rest) was not superior to a lenient rate control strategy $(<110 \mathrm{bpm}$ at rest). ${ }^{6}$ The mean heart rates of our study patients were in the same range as in RACE-II, where the mean heart rate achieved was $93 \mathrm{bpm}$ and $76 \mathrm{bpm}$ in the lenient and strict rate control arm, respectively. While one of the few randomised trials in this field, the sample size in RACE-II was relatively small, the primary endpoint included heterogeneous outcomes, and patients with a history of stroke were excluded, ${ }^{6}$ such that our study may provide some reassurance with regard to the generalisability of these findings.

\section{Strengths and limitations}

Strengths of this analysis include the relatively large sample size of contemporary patients with $\mathrm{AF}$, a limited amount of missing data and a relatively long and complete follow-up. However, there are some potential limitations that should be considered when interpreting our data. First, as in any observational study, we cannot assess causality and residual confounding may persist even after extensive multivariable adjustment. Moreover, some residual confounding due to change in confounders during follow-up may also exist, but given that adjustment for the covariates at baseline had little effect on the results, we do not consider that any such confounding would have significantly altered these results. Second, heart rate thresholds ( $<60$ and $\geq 100 \mathrm{bpm}$ ) were chosen to represent commonly used definitions of bradycardia and tachycardia. Our study was not sufficiently powered to determine whether more extreme heart rates are significantly associated with adverse outcome events. In addition, many $\mathrm{AF}$ patients with more extreme heart rates are symptomatic and require interventions anyway. Third, the heart rate was derived from one $10 \mathrm{~s}$ ECG strip at baseline. While this is the current clinical standard in most stable patients with $\mathrm{AF}$, it is possible that a more detailed assessment of the heart rate using longer recording times (eg, 24-hour Holter recording) may provide different associations with clinical outcomes in this population. It is also possible that related parameters such as heart rate variability may improve risk stratification, and further studies are needed in this area. Nevertheless, current clinical practice still heavily relies on short heart rate assessments.

\section{CONCLUSIONS}

In conclusion, in this large cohort of patients with prevalent $\mathrm{AF}$, heart rate was not associated with heart failure hospitalisation, stroke or systemic embolism and death. These data are in line with recommendations that strict heart rate control is not needed in most stable outpatients with $\mathrm{AF}$.

\section{Author affiliations}

${ }^{1}$ Division of Cardiology, Regional Hospital of Lugano, Repubblica e Cantone Ticino Ente Ospedaliero Cantonale, Lugano, Ticino, Switzerland

${ }^{2}$ Population Health Research Institute, McMaster University, Hamilton, Ontario,

Canada

${ }^{3}$ Clinical Sciences, Lund University, Malmö, Sweden

${ }^{4}$ Division of Cardiology, University Hospital Basel, Basel, Switzerland

${ }^{5}$ Cardiovascular Research Institute Basel, University Hospital Basel, Basel, Switzerland

${ }^{6}$ Unit of Clinical Epidemiology, Repubblica e Cantone Ticino Ente Ospedaliero Cantonale, Bellinzona, Ticino, Switzerland

${ }^{7}$ Cardiology Division, Heart Center, Luzerner Kantonsspital, Luzern, Luzern, Switzerland

${ }^{8}$ Division of Cardiology, Ospedale San Giovanni, Repubblica e Cantone Ticino Ente Ospedaliero Cantonale, Bellinzona, Ticino, Switzerland

${ }^{9}$ Faculty of Biomedical Sciences, Università della Svizzera Italiana, Lugano, Ticino, Switzerland

${ }^{10}$ Division of Cardiology, Fondazione Cardiocentro Ticino, Lugano, Ticino,

Switzerland

${ }^{11}$ Institute of Primary Health Care, University of Bern, Bern, Switzerland

${ }^{12}$ Department of General Medicine, Inselspital University Hospital Bern, Bern, Switzerland

${ }^{13}$ Department of Medicine, Baden Cantonal Hospital, Baden, Aargau, Switzerland

${ }^{14}$ Center for Molecular Cardiology, University of Zurich, Zurich, Switzerland

Twitter Linda S B Johnson @ljsMD

Acknowledgements We would like to thank all the patients who participated to the study. 
Contributors GM and DC conceived this analysis. GM, SB, SA, MLDP, PAMM, RK, MDV, CSZ, AA, GC, NR, MRB, JHB, MK, SO and DC contributed to data collection. LSBJ and DC planned the statistical methodology, which LSBJ performed and reported. GM and LSBJ drafted the manuscript. All coauthors contributed critical revisions to the manuscript. All authors approved the final version of the manuscript. GM, LSBJ and DC are responsible for the overall content of the work as guarantors.

Funding BEAT-AF has been supported by the Swiss National Science Foundation (PP00P3_159322), the Swiss Heart Foundation, the University Hospital of Basel, Roche Diagnostics, Boehringer Ingelheim, Sanofi-Aventis, Merck Sharp \& Dome, Bayer, Daiichi-Sankyo and Pfizer/Bristol-Myers Squibb. Swiss-AF has been supported by grants of the Swiss National Science Foundation (grant numbers 33CS30_148474 and 33CS30_177520), the Foundation for Cardiovascular Research Basel (FCVR), the University of Basel and Roche Diagnostics. DC holds a McMaster University Department of Medicine Mid-Career Research Award. LSBJ is supported by governmental funding within the Swedish National Health Services, as well as by the Swedish Society of Medicine, the Swedish Heart and Lung Foundation, the Swedish Heart and Lung Association and the Bergqvist Foundation.

Competing interests Disclosures/conflicts of interest outside of the submitted work, are reported within the manuscript. GM has received consultant fees for taking part to advisory boards from Novartis and Astra Zeneca outside of the submitted work. RK receives institutional grants from Abbott, BiosenseWebster, Boston-Scientific, Biotronik, Medtronic and Sis-Medical. CSZ reports a research grant from Medtronic and honoraria from Vifor Pharma and Novartis. AA has served as consultant to Boston Scientific, Backbeat, Biosense Webster, Cairdac, Corvia, Microport CRM, EPD-Philips and Radcliffe Publisher; he received speaker fees from Boston Scientific, Medtronic, and Microport; he participates in clinical trials sponsored by Boston Scientific, Medtronic, EPD-Philips; and he has intellectual properties with Boston Scientific, Biosense Webster, and Microport CRM. JHB reports grants from the Swiss National Foundation of Science, The Swiss Heart Foundation. MK has served on the speaker's bureau for Boston Scientific, St. Jude Medical and Biotronik; he has received lecture/consulting fees from Sorin, Boehringer Ingelheim, Bayer, Sanofi Aventis, Novartis, Medtronic, Pfizer-BMS and has received unrestricted grants from Bayer and Pfizer-BMS; he is a proctor for Medtronic (Cryoballoon). DC has received consultant/speaker fees from Servier Canada and Roche Diagnostics, Switzerland, outside of the submitted work.

\section{Patient consent for publication Not required.}

Ethics approval The study comply with the Declaration of Helsinki. The research protocol has been approved by the locally appointed ethics committee.

Provenance and peer review Not commissioned; externally peer reviewed.

Data availability statement No data are available.

Open access This is an open access article distributed in accordance with the Creative Commons Attribution Non Commercial (CC BY-NC 4.0) license, which permits others to distribute, remix, adapt, build upon this work non-commercially, and license their derivative works on different terms, provided the original work is properly cited, appropriate credit is given, any changes made indicated, and the use is non-commercial. See: http://creativecommons.org/licenses/by-nc/4.0/.

\section{ORCID iDs}

Giorgio Moschovitis http://orcid.org/0000-0002-4043-8061

Linda S B Johnson http://orcid.org/0000-0002-2249-8220

Stefanie Aeschbacher http://orcid.org/0000-0001-8134-2421

Giulio Conte http://orcid.org/0000-0003-2248-3456

\section{REFERENCES}

1 Krijthe BP, Kunst A, Benjamin EJ, et al. Projections on the number of individuals with atrial fibrillation in the European Union, from 2000 to 2060. Eur Heart J 2013;34:2746-51.
2 Wang TJ, Larson MG, Levy D, et al. Temporal relations of atrial fibrillation and congestive heart failure and their joint influence on mortality: the Framingham heart study. Circulation 2003;107:2920-5.

3 Wolf PA, Mitchell JB, Baker CS, et al. Impact of atrial fibrillation on mortality, stroke, and medical costs. Arch Intern Med 1998;158:229-34.

4 Conen D, Chae CU, Glynn RJ, et al. Risk of death and cardiovascular events in initially healthy women with new-onset atrial fibrillation. JAMA 2011;305:2080-7.

5 Wyse DG, Waldo AL, DiMarco JP, et al. A comparison of rate control and rhythm control in patients with atrial fibrillation. N Engl $\mathrm{J}$ Med 2002;347:1825-33.

6 Van Gelder IC, Groenveld HF, Crijns HJGM, et al. Lenient versus strict rate control in patients with atrial fibrillation. $N$ Engl $J$ Med 2010;362:1363-73.

7 Van Gelder IC, Wyse DG, Chandler ML, et al. Does intensity of ratecontrol influence outcome in atrial fibrillation? an analysis of pooled data from the race and affirm studies. Europace 2006;8:935-42.

8 Andrade JG, Roy D, Wyse DG, et al. Heart rate and adverse outcomes in patients with atrial fibrillation: a combined affirm and AF-CHF substudy. Heart Rhythm 2016;13:54-61.

9 Kannel WB, Kannel C, Paffenbarger RS, et al. Heart rate and cardiovascular mortality: the Framingham study. Am Heart $J$ 1987;113:1489-94.

10 Fox K, Ford I, Steg PG, et al. Heart rate as a prognostic risk factor in patients with coronary artery disease and left-ventricular systolic dysfunction (beautiful): a subgroup analysis of a randomised controlled trial. Lancet 2008;372:817-21.

11 Tverdal A, Hjellvik V, Selmer R. Heart rate and mortality from cardiovascular causes: a 12 year follow-up study of 379,843 men and women aged 40-45 years. Eur Heart J 2008;29:2772-81.

12 Hindricks G, Potpara T, Dagres N, et al. 2020 ESC guidelines for the diagnosis and management of atrial fibrillation developed in collaboration with the European association for Cardio-Thoracic surgery (EACTS). Eur Heart J 2021;42:373-498.

13 Zimny M, Blum S, Ammann P, et al. Uptake of non-vitamin K antagonist oral anti coagulants in patients with atrial fibrillation - a prospective cohort study. Swiss Med Wkly 2017;147:w14410.

14 Blum S, Muff C, Aeschbacher S, et al. Prospective assessment of sex-related differences in symptom status and health perception among patients with atrial fibrillation. J Am Heart Assoc 2017:6:e005401.

15 Conen D, Rodondi N, Mueller A, et al. Design of the Swiss atrial fibrillation cohort study (Swiss-AF): structural brain damage and cognitive decline among patients with atrial fibrillation. Swiss Med Wkly 2017;147:w14467-w..

16 Conen D, Rodondi N, Müller A, et al. Relationships of overt and silent brain lesions with cognitive function in patients with atrial fibrillation. J Am Coll Cardiol 2019;73:989-99.

17 European Heart Rhythm Association, European Association for Cardio-Thoracic Surgery, Camm AJ, et al. Guidelines for the management of atrial fibrillation: the task force for the management of atrial fibrillation of the European Society of cardiology (ESC). Eur Heart J 2010;31:2369-429.

18 Fine JP, Gray RJ. A proportional hazards model for the Subdistribution of a competing risk. J Am Stat Assoc 1999;94:496-509.

19 Kirchhof P, Camm AJ, Goette A, et al. Early rhythm-control therapy in patients with atrial fibrillation. N Engl J Med 2020;383:1305-16.

20 Levy T, Walker S, Mason M, et al. Importance of rate control or rate regulation for improving exercise capacity and quality of life in patients with permanent atrial fibrillation and normal left ventricular function: a randomised controlled study. Heart 2001;85:171-8.

21 Pandey A, Kim S, Moore C, et al. Predictors and Prognostic Implications of Incident Heart Failure in Patients With Prevalent Atria Fibrillation. JACC Heart Fail 2017:5:44-52.

22 Ponikowski P, Voors AA, Anker SD, et al. 2016 ESC Guidelines for the diagnosis and treatment of acute and chronic heart failure: The Task Force for the diagnosis and treatment of acute and chronic heart failure of the European Society of Cardiology (ESC)Developed with the special contribution of the Heart Failure Association (HFA) of the ESC. Eur Heart J 2016;37:2129-200. 